\title{
DIAGNÓSTICO DA ATETOSE POR TÉCNICAS DE NEUROIMAGEM: ESTUDOS DE ANATOMIA E PARALISIA CEREBRAL
}

\author{
DIAGNOSIS OF ATHETOSIS THROUGH NEUROIMAGE TECHNIQUES: EMPHASIS \\ ON MAGNETIC RESONANCE AS PROOF IN ANATOMY STUDIES
}

\begin{abstract}
André Luiz dos Santos ${ }^{1}$
RESUMO: A ocorrência de Paralisia Cerebral pode desencadear numa criança alterações do movimento, de postura, do equilíbrio, da coordenação e do tônus muscular, prejudicando o desenvolvimento neuropsicomotor normal. Atetose é um termo que descreve um tipo particular de movimento comum a pessoas com condições como a doença de Huntington e outras que afetam o sistema nervoso central. Para o diagnóstico correto da patologia, vários exames podem ser utilizados, no entanto, os exames de neuroimagem, como ultrassonografia transcraniana (UTC), Ressonância Magnética (RM) ou tomografia computadorizada (TC), para verificar se há tumores ou evidência de acidente vascular cerebral, são as técnicas mais utilizadas para um diagnóstico mais preciso. Diante de tal contexto, este trabalho tem como objetivo fazer uma revisão bibliográfica sobre a Avaliação da Atetose através de Neuroimagem, principalmente o uso da Ressonância Magnética. É possível concluir que a ressonância magnética é uma poderosa prova para estudos de anatomia e para avaliar as alterações que ocorrem com o desenvolvimento do cérebro.
\end{abstract}

Palavras-chave: Diagnóstico. Paralisia cerebral. Sistema nervoso central.

ABSTRACT: The occurrence of Cerebral Palsy can trigger changes in children movement, body posture, physical balance, coercion and muscle tone. Any of the above cases could end up damaging normal neurological and psychomotor development. "Athetosis "is the term that describes a particular type of movement, common to people with Huntington's Disease and others who affect the central nervous system. For obtein the correct diagnosis we can use severals exams, however, Neuroimaging tests, such as Ultrasound, Magnetic Resonance Imaging or Computed Tomography, to check for tumor or evidence of strokes, these are the techniques commonly used for the most accurate result. Given this context, the work cited aims to make a literature review on the "Evaluation of Athetosis" through neurimaging, especially the use of Magnetic Resonance. We then conclude that the latter examination is powerful evidence for anatomy studies, as well as for assessing changes that occur with brain development.

Keywords: Diagnosis. Cerebral Palsy. Central nervous system.

\footnotetext{
' Biólogo e Pedagogo, associado profissionalmente ao Banco de Tecidos do Instituto Nacional de Traumatologia e Ortopedia(INTO), como Servidor na Fundação Saúde do Rio de Janeiro (FSERJ). Associado academicamente ao Instituto Brasileiro de Medicina de Reabilitação (IBMR). Email: lopanrio@hotmail.com. Orcidid: https://orcid.org/oooo-ooo2-5638-1581.
} 


\section{INTRODUÇÃO}

Um dos problemas neurológicos mais frequentes e importantes em crianças é a paralisia cerebral (PC), e esta acarreta deficiências físicas e/ou mentais. A PC, também denominada encefalopatia crônica não-progressiva da infância, é consequência de uma lesão estática, ocorrida no período pré, peri, ou pós-natal que afeta o sistema nervoso central em fase de maturação estrutural e funcional (ROTTA, 2002).

Epidemiologicamente há uma incidência nos países desenvolvidos em torno de 2 ocorrências para cada rooo indivíduos nascidos vivos, números persistentes. Existem evidências científicas de que a incidência da paralisia cerebral aumenta em crianças que nascem prematuramente $e$ inclusive por este motivo, nascem com baixo peso ao nascimento (MARANHÃO, 2005).

Maranhão ainda destaca que (2005) nota-se grande variabilidade nas sequelas do nascimento com a paralisia cerebral, podendo acontecer de uma leve monoplegia acompanhada de uma capacidade intelectual normal até hipertonia e hiperreflexia do tônus muscular associada ao retardo mental. Além do potencial citado, as crianças com paralisia cerebral podem ainda apresentar uma outra deficiência por consequência da lesão no sistema nervoso central, como afetação do desenvolvimento cognitivo, deficiências na visão e na audição, problemas comportamentais e doenças crônicas sistêmicas associadas, tais como ortopédicas, gastrintestinais e respiratórias.

Estudo recente abrangendo o período de 1970 até 1999 com amostragem de 70824 indivíduos recém-nascidos tabulou 2,3 pacientes com paralisia cerebral de etiologia pré e perinatal para cada ıoo pacientes recém-nascidos. Importante verificar também que a maior parte dos pacientes nasceu abaixo do peso (<2500 g). A paralisia cerebral pode ter causas pós-natais, tais como a meningite, encefalite viral, hidrocefalia, trauma, oclusão de veias e artérias encefálicas e lesões cirúrgicas. Destaca-se no estudo que causas de paralisia cerebral mais comuns após o nascimento podem ser as infecções (50\%), alterações vasculares (20\%) e trauma (I8\%) (MARANHÃO, 2005).

A PC pode ser classificada de acordo com a região cerebral acometida, conforme a severidade do comprometimento, o tipo de desordem do movimento ou pela distribuição topográfica da lesão (WESTCOTT; GOULET, 2008). Uma das causas da PC é a icterícia 
neonatal patológica, em que ocorre a impregnação dos núcleos da base no cérebro do bebê pela bilirrubina indireta. As sequelas clássicas da encefalopatia crônica por bilirrubina (Kernicterus) incluem distúrbios do movimento como atetose, distonia e coeroatetose, surdez neurossensorial (BHUTANI et al.,2004; MAISELS, 2007).

A atetose é uma disfunção do movimento. É caracterizada por movimentos involuntários de contorção. Esses movimentos podem ser contínuos, lentos e contínuos. Eles também podem dificultar a manutenção de uma postura simétrica e estável. Com a atetose, as mesmas regiões do corpo são afetadas repetidamente. Isso geralmente inclui mãos, braços e pés. $\mathrm{O}$ pescoço, o rosto, a língua e o tronco também podem estar envolvidos (HOLLAND, 2019).

Embora a atetose possa ser contínua, pode piorar com as tentativas de controlar o movimento. Por exemplo, se uma pessoa com a condição tentar digitar em um teclado de computador, ela poderá ter extrema dificuldade em controlar onde seus dedos pousam e por quanto tempo permanecem (HOLLAND, 2019).

A atetose é um sintoma de um distúrbio, raramente é um distúrbio por si só. É por isso o médico trabalhará para diagnosticar a causa subjacente dos problemas de movimento. Uma vez que a causa é identificada, o médico pode tratá-la. Isso deve ajudar a eliminar ou limitar os sintomas da atetose (HOLLAND, 2019).

Para o diagnóstico correto da patologia, vários exames podem ser utilizados, no entanto, os exames de neuroimagem, como ultrassonografia transcraniana (UTC), Ressonância Magnética (RM) ou tomografia computadorizada (TC), para verificar se há tumores ou evidência de acidente vascular cerebral, são as técnicas mais utilizadas para um diagnóstico mais preciso. Diante de tal contexto, tal trabalho tem como objetivo fazer uma revisão bibliográfica sobre a Avaliação da Atetose através de Neuroimagem, principalmente o uso da Ressonância Magnética.

\section{ATETOSE}

Athetose", do grego athetos, que significa "sem posição fixa", é um distúrbio do movimento descrito pela primeira vez por Hammond em I871 . O termo descreveu movimentos contínuos lentos e irregulares das extremidades distais. Em 1983, Foley 
definiu a síndrome atetóide como "um distúrbio não progressivo, mas em evolução, devido a danos nos gânglios basais do cérebro a longo prazo, comprometimento dos reflexos posturais, movimentos involuntários arrítmicos e disartria, movimentos oculares" (PRZEKOP; TERENCE, 2OII).

Atetose é um termo que descreve um tipo particular de movimento comum a pessoas com condições como a doença de Huntington e outras que afetam o sistema nervoso central. O movimento atético é lento e sinuoso, e afeta principalmente os braços, pernas, mãos e pés. As pessoas com essa condição também podem experimentar outros tipos de movimentos atípicos, como a coréia, que causa movimentos bruscos involuntários, movimentos rápidos das mãos e dos pés (PORTAL SÃO FRANCISCO,2019).

Uma década depois, a "síndrome atetoide" foi substituída por "paralisia cerebral discinética”. A lesão nos gânglios da base por vários mecanismos, incluindo asfixia, trauma, acidente vascular cerebral perinatal e kernicterus que é conhecida por causar atetose relacionada ao nascimento. Kernicterus descreveu originalmente a neuropatologia da lesão cerebral induzida por bilirrubina, onde os núcleos profundos do cérebro ficam amarelados. Kernicterus agora descreve os aspectos clínicos da encefalopatia crônica por bilirrubina, que incluem um distúrbio do movimento extrapiramidal, perda auditiva neurossensorial, diminuição do olhar para cima e displasia do esmalte dental (PRZEKOP; TERENCE, 20II).

O movimento atético pode ser causado de várias maneiras. As causas mais comuns são paralisia cerebral, encefalite, tumores cerebrais, derrame, febre reumática, lúpus eritematoso sistêmico e traumatismo cranioencefálico. O Kernicterus, um tipo grave de icterícia que ocorre raramente em recém-nascidos, pode levar ao tipo de dano cerebral que pode causar movimentos atópicos. Além disso, a atetose pode se desenvolver como um efeito colateral dos medicamentos antipsicóticos, ou como resultado de uma overdose de anfetamina ou envenenamento por monóxido de carbono (PORTAL SÃO FRANCISCO, 2019). 


\section{DIAGNÓSTICO POR NEUROIMAGEM}

Segundo Margotto (2019), na era pré-ressonância magnética, somente os estudos de pós-mortem podiam confirmar kernicterus. Crucial para o diagnóstico é o reconhecimento do tipo de lesão neuronal seletiva envolvendo o globo pálido e os núcleos subtalâmicos com residual gliose, contrário do que ocorre na lesão hipóxico-isquêmica (envolve o putamen e o tálamo).

Um estudo analisou os achados de neuroimagem de ultra-som e Ressonância Magnética de onze crianças de diferentes idades gestacionais com severa hiperbilirrubinemia e/ou sinais de encefalopatia bilirrubínica.

Os autores não encontraram uma relação consistente entre anormalidades das imagens recentes e o resultado motor, mas em imagens posteriores todas as crianças com tiveram mudanças clássicas no globo pálido. Percebe-se assim a importância destes exames no diagnóstico de tal patologia (GKOLTSIOU et al., 2008).

$\mathrm{Na}$ ciência neurológica a ultrassonografia transcraniana (UTC) é um método de neuroimagem que parte da aplicação de pulsos ultrassônicos obtendo imagens em tons de cinza do cérebro. Através da aplicação deste valioso método obtém-se a acurácia necessária para diagnóstico de variadas doenças, inclusive no diagnóstico da doença de Parkinson e no diagnóstico diferencial de outras doenças do movimento (FERNANDES; BERG, 2015; BERG; GODAU; WALTER, 2008).

A literatura radiológica brasileira vem, recentemente, ressaltando a importância dos exames de RM no aprimoramento do diagnóstico do sistema nervoso central (ALFENAS; NIEMEYER; BAHIA, 2014; BARBOSA; SANTOS; SALMON, 2015). Kernicterus, também conhecido como encefalopatia bilirrubínica, é uma complicação rara de hiperbilirrubinemia na infância, ocorrendo quando são atingidos níveis séricos superiores a $20 \mathrm{mg} / \mathrm{dL}$ no neonato a termo ou até mesmo valores menores em prematuros, resultando em depósito de bilirrubina nos globos pálidos, núcleos subtalâmicos, hipocampo, putâmen, tálamo e nervos cranianos (principalmente III, IV e VI) (RIBEIRO et al., 20I6).

Figura I 

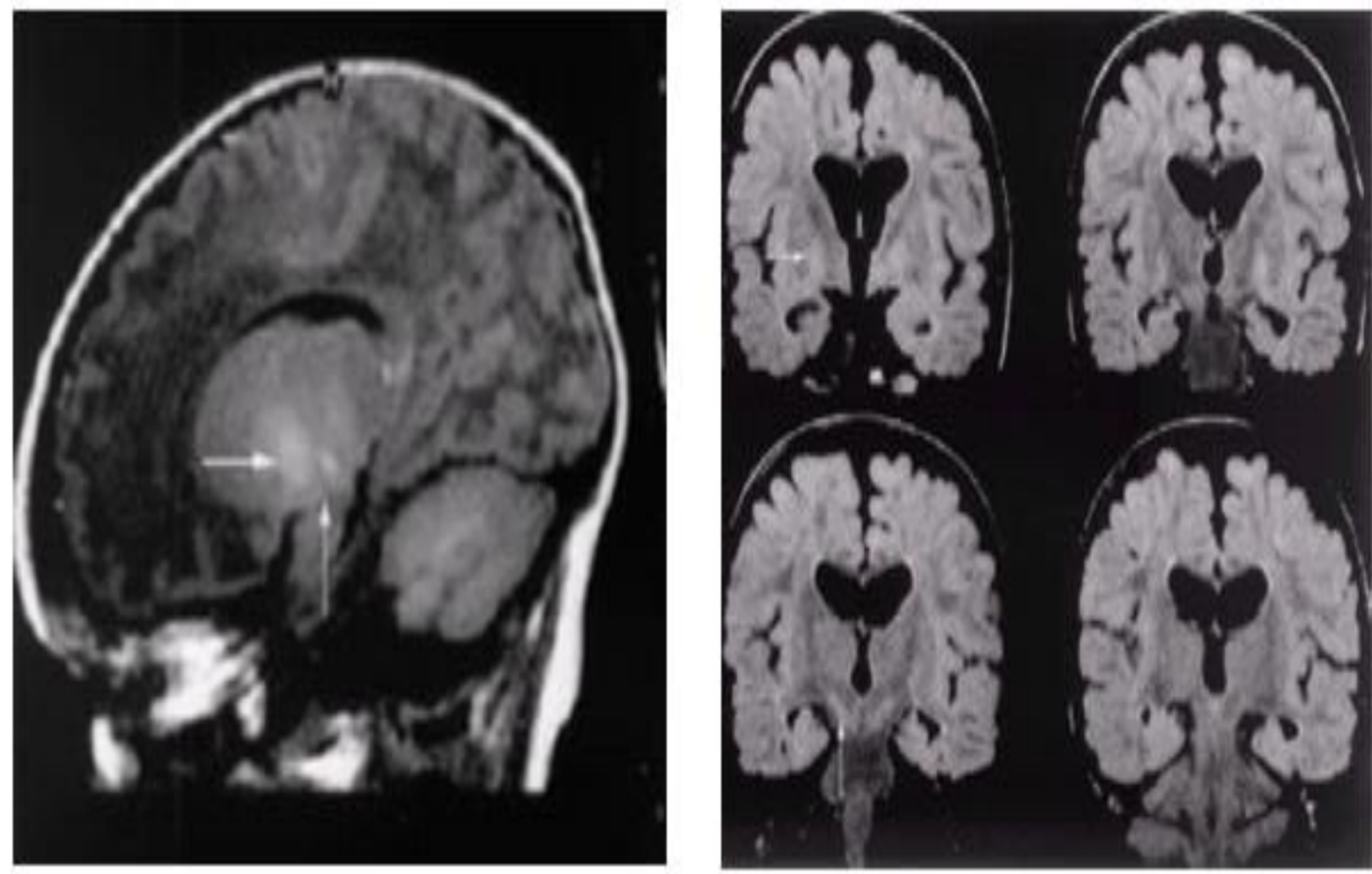

Fig I: RM (TI). Lesão nos núcleos subtalâmicos. (A) Paciente 8 aos I2 dias evidenciando sinal de alta intensidade anormal no globo pálido (seta horizontal) e subtálamo (seta vertical). (B): RM (fluid attenuated inversion recovery-FLAIR-) no paciente 3, aos 22 meses, evidenciando sinal de alta intensidade anormal subtalâmico (seta vertical) e globo pálido (seta horizontal). Fonte: Adaptada de GOVAERT et al., (2003)

Os achados de imagem na RM no kernicterus são caracterizados por hipersinal nas sequências ponderadas em $\mathrm{T}_{\mathrm{I}}$ nos globos pálidos, evoluindo cronicamente para uma substituição do hipersinal em TI por um hipersinal permanente em T2 e FLAIR, tanto nos globos pálidos quanto nos núcleos subtalâmicos, de aspecto bilateral e simétrico (PARASHARI et al., 2009; RIBEIRO et al., 2016; GOVAERT et al., 2003), correspondendo às áreas de deposição preferencial da bilirrubina não conjugada, configurando o kernicterus crônico, como no caso apresentado (Ver figura I).

Segundo Ribeiro e colaboradores (2016), foi possível observar através da Ressonância magnética (RM) do crânio que mostrou hipersinal nas sequências FLAIR e 
T2, bilateral e simétrico, acometendo globos pálidos e núcleos subtalâmicos, sem determinar efeito de massa, não apresentando restrição à difusão e sem evidência de realce pelo gadolínio (Figura 2). Tais achados de imagem, associados à história clinicolaboratorial, confirmaram o diagnóstico de kernicterus crônico.

\section{Imagem 2}
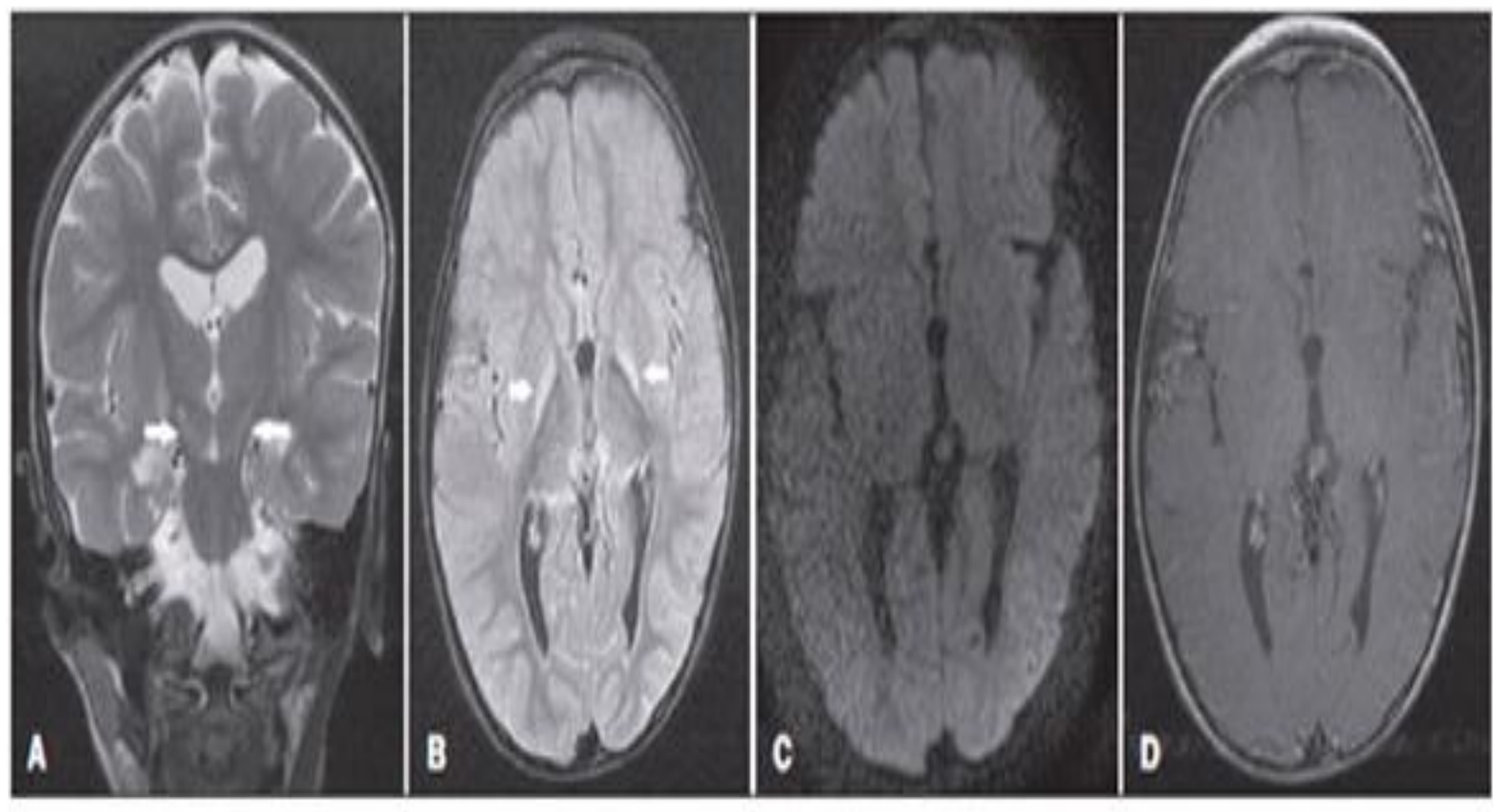

Figura 2. (A) Imagem coronal na sequência $T_{2}$ mostrando hipersinal nos núcleos subtalâmicos (setas), bilaterais e simétricos, sem efeito de massa. (B) Imagem axial na sequência FLAIR exibindo hipersinal nos globos pálidos (setas), bilaterais e simétricos. (C) Imagem axial na sequência difusão não demonstrando restrição à difusão. (D) Imagem axial na sequência $T_{I}$ sem evidência de realce pelo gadolínio. Fonte: Adaptada de Ribeiro et al., (2016)

Segundo Rose e Vassar (2015), lesões cerebrais identificadas por Ressonância Magnética após hiperbilirrubinemia extrema foram associadas à PC discinética. Técnicas de imagem mais recentes, como imagens de tensores de difusão ou a tomografia computadorizada de emissão de fóton único, permite a quantificação de lesões mais sutis 
da substância branca após a exposição presumida à bilirrubina não ligada e pode explicar distúrbios mais sutis do movimento.

Hayashi e colaboradores (199I) dividiram os casos de paralisia cerebral atetóide em dois grupos: I) Grupo globo pálido e núcleos subtalâmicos (relacionado a severa hiperbilirrubinemia) e 2) grupo talamo-putamen (relacionado a asfixia neonatal). Os pacientes do grupo talamo-putamen mostraram menor habilidade mental e apresentaram mais convulsões intratáveis do que o grupo globo pálido. No grupo globo pálido, a espasticidade rígida foi demonstrada com freqüência, assim como movimentos atetóticos.

\section{CONCLUSÃO}

Deste modo, é possível concluir que a ressonância magnética é uma prova importante para estudos de anatomia e para avaliar as alterações que ocorrem com o desenvolvimento do cérebro. O kernicterus pode ser suspeito no período neonatal com dados clínicos específicos, laboratoriais e de imagem. A ressonância magnética tem um valor crucial, mas o sinal muda em $\mathrm{T}_{\mathrm{I}}$ e $\mathrm{T}_{2}$. Os estudos confirmam os achados de anormalidades do sinal em $T_{1}$ e em $T_{2}$ na ressonância magnética feita mais precocemente. O padrão de lesão precoce pode ser identificada na ultra-sonografia cerebral.

\section{REFERÊNCIAS}

ALFENAS, R.; NIEMEYER, B.; BAHIA, P.R.V, et al. Parry-Romberg syndrome: findings in advanced magnetic resonance imaging sequences - case report. Radiol Bras. 2014;47:186-8.

BARBOSA, J.H.O.; SANTOS, A.C.; SALMON, C.E.G. Susceptibility weighted imaging: differentiating between calcification and hemosiderin. Radiol Bras. 2015;48:93IOO.

BERG, D.; GODAU, J.; WALTER, U. Transcranial sonography in movement disorders. Lancet Neurol 2008;7:1044-55 
BHUTANI VK, JOHNSON LH, MAISELS MJ, NEWMAN TB, PHIBBS C, STARK AR, YEARGIN-ALLSOPP M. Kernicterus: Epidemiological Strategies for its Prevention through Systems-based Approaches. J Perinatol. 2004; 24: 650-662.

FERNANDES, R.C.L.; BERG, D. Parenchymal imaging in movement disorders. In: A. Alonso; M.G. Hennerici; S. Meairs (Eds.) Frontiers of Neurology and Neuroscience - vol 36: Translational Neurosonology, p.7I-82. Ist Ed. Basel, S Karger AG, 2015; DOI I0.1159/000366238.

Gkoltsiou, K.; Tzoufi, M.; Counsell, S.; Rutherford, M.; Cowan, F. Serial brain MRI and ultrasound findings: Relation to gestation age, bilirrubin level, neonatal neurologic status and neurodevelopmental outcome in infants at risk of kernicterus. Early Hum Dev. 2008; 84(12): 829-838.

GOVAERT, P.; LEQUIN, M.; SWARTE, R. et al. Changes in globus pallidus with (pre)term kernicterus. Pediatrics. 2003;112(6 Pt I):1256-63.

HAYASHI, M. et al. Clinical and neuropathological findings in severe athetoid cerebral palsy: a comparative study og globo-Luysian and thalamo-putaminal groups. Brain Dev 1991;13:47-51

HOLLAND, K. O que é atetose? 2019. Disponível em < https://www.healthline.com/health/athetosis $>$. Acessado em: 20 de Out de 2019

MARANHAO, Március Vinícius M. Anestesia e paralisia cerebral. Rev. Bras. Anestesiol., Campinas, v. 55, n. 6, p. 680-702, Dec. 2005. Disponível em: $<$ http://www.scielo.br/scielo.php?script=sci_arttext\&pid=Soo34-

7094200500o6ooor2\&lng=en\&nrm=iso $>$ Acessado em: i6 de Feb 2021. https://doi.org/10.1590/So034-70942005000600o12.

MARGOTTO,P.R. Kernicterus, ainda um Desafio. Disponível em 〈www.paulomargotto.com.br $>$. Acessado em: 20 de Out de 2019

MAISELS, M.J. Icterícia. In: Macdonald MG, Seshia MMK, Mullett MD. Fisiopatologia e tratamento do Recém-nascido. 6ª edição, Rio de Janeiro: Guanabara Koogan, 2007. p. 703777 .

PARASHARI, U.C.; SINGH, R.; YADAV R, et al. Changes in the globus pallidus in chronic kernicterus. J Pediatr Neurosci. 2009;4:117-9. 
PORTAL SÃO FRANCISCO. Atetose. 2019. Disponível em

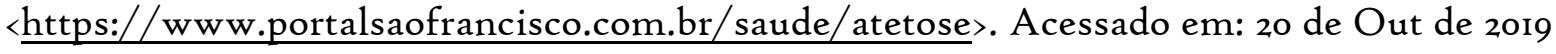
PRZEKOP, A.; SANGER, T. D. Chapter 30 - Birth-related syndromes of athetosis and kernicterus. Handbook of Clinical Neurology, V Ioo, 2011, Pages 387-395. Disponível em $<$ https://www.sciencedirect.com/science/article/pii/B9780444520142000306 $>$. Acessado em: 20 de Out de 2019

RIBEIRO, B. N. de F.; LIMA, G. de A.; Ventura, N.; GASPARETTO, E. L.; Marchiori, E. Kernicterus crônico: achados na ressonância magnética. Radiol Bras. 2016 Nov/Dez;49(6):406-413.

Disponível em

http://www.rb.org.br/detalhe_artigo.asp?id=2797\&idioma=Portugues $>$. Acessado em: 20 de Out de 2019

ROSE, J.; VASSARAC, R. Movement disorders due to bilirubin toxicity. Seminars in Fetal and Neonatal Medicine V 20, Issue I, February 2015, Pages 20-25. Disponível em:

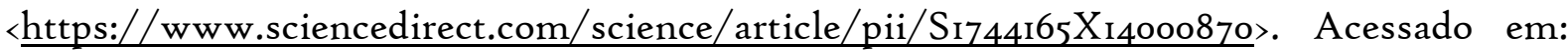
20 de Out de 2019

ROTTA, N.T. Paralisia cerebral, novas perspectivas terapêuticas. J Pediatr (Rio J). 2002; 78(Supl.I):s48-s54.

TURKEL, S.B.; MILleR, C.A.; GUTTENBERG, M.E, et al. A clinical pathologic reappraisal of kernicterus. Pediatrics. 1982;69:267-72.

WESTCOTT, S.L.; GOULET, C. Sistema Neuromuscular: estruturas, funções, diagnóstico e avaliação. In: Effgen SK. Fisioterapia em Pediatria: atendendo às necessidades das crianças. Rio de Janeiro: Guanabara Koogan, 2007, p.159-208. 